SINP-TNP/07-21

\title{
Universal near-horizon conformal structure and black hole entropy
}

\author{
Sayan K. Chakrabarti] \& Kumar S. Gupta 2 \\ Theory Division \\ Saha Institute of Nuclear Physics \\ 1/AF Bidhannagar, Calcutta - 700064, India \\ Siddhartha Sen ${ }^{3}$ \\ School of Mathematical Sciences \\ UCD, Belfield, Dublin 4, Ireland \\ and \\ Department of Theoretical Physics \\ Indian Association for the Cultivation of Science \\ Calcutta - 700032, India
}

\begin{abstract}
It is shown that a massless scalar probe reveals a universal near-horizon conformal structure for a wide class of black holes, including the BTZ. The central charge of the corresponding Virasoro algebra contains information about the black hole. With a suitable quantization condition on the central charge, the CFT associated with the black hole in our approach is consistent with the recent observation of Witten, where the dual theory for the BTZ in the AdS/CFT framework has been identified with the construction of Frenkel, Lepowsky and Meurman. This CFT admits the Fischer-Griess monster group as its symmetry. The logarithm of the dimension of a specific representation of the monster group has been identified by Witten as the entropy of the BTZ black hole. Our algebraic approach shows that a wide class of black holes share the same near-horizon conformal structure as that for the BTZ. With a suitable quantization condition, the CFT's for all these black holes in our formalism can be identified with the FLM model, although not through the AdS/CFT correspondence. The corresponding entropy for the BTZ provides a lower bound for the entropy of this entire class of black holes.
\end{abstract}

August 2007

PACS : 04.70.Dy

\footnotetext{
${ }^{1}$ Email: sayan.chakrabarti@saha.ac.in

${ }^{2}$ Email: kumars.gupta@saha.ac.in

${ }^{3}$ Email: sen@maths.ucd.ie
} 


\section{Introduction}

Conformal field theory plays a central role in the analysis of various quantum aspects of black holes [1, 2, 3. In particular, for spaces with negative cosmological constant, the AdS/CFT duality [4, 5] has led to remarkable insights in black hole physics. This duality, which encodes the holographic principle [6, 7, 8] for AdS spaces, provides a successful derivations of black hole entropy, consistent with the Bekenstein-Hawking expression.

Recently, Witten [9] has used the AdS/CFT duality to obtain the entropy of a quantum $2+1$ dimensional BTZ black hole [10, 11]. The BTZ black hole is associated with a Virasoro algebra whose classical central charge, obtained using the method of Brown and Henneaux [12], is a continuous quantity. It has however been argued that in the dual description the central charge $c$ is not continuous, but is quantized as $24 k$, where $k$ is a positive integer. This is consistent with the ChernSimons description [13, 14] of BTZ for a suitable choice of the gauge group. For $k=1$, Witten [9] has identified the corresponding dual theory with the $c=24$ holomorphic CFT obtained by Frenkel, Lepowsky and Meurman (FLM) 15. The FLM CFT describes only gravitational degrees of freedom and is not associated with any gauge fields. In addition, the $c=24$ FLM CFT is conjectured to be unique [16] and admits a symmetry given by the Fischer-Griess monster group. Using this symmetry, Witten calculated the entropy of the quantum BTZ black hole as the logarithm of the dimension of a specific representation of the monster group relevant for the FLM CFT. The result obtained is in reasonable agreement with the semiclassical value for the Bekenstein-Hawking entropy.

Another approach which has been very useful in black hole entropy analysis is based on the existence of a CFT in the near-horizon region of black holes [2, 17, 18, 19]. The analysis here is also based on Brown and Henneaux's approach [12], suitably adapted to the near-horizon region. This approach has led to the derivation of entropy for BTZ black hole as well as for Schwarzschild black holes in arbitrary dimensions and the results obtained are consistent with the Bekenstein-Hawking formula.

In a previous paper we have shown that the near-horizon conformal structure can also be revealed by probing the black hole geometry with a massless scalar probe [20. Using the zero mode of a massless scalar field, this approach was shown to be consistent with the existence of a Virasoro algebra in the near-horizon region of $3+1$ dimensional Schwarzschild black hole. Our approach also predicted the logarithmic correction to the Bekenstein-Hawking entropy [21]. Similar scalar field probe can also be used to analyze particle production in black hole backgrounds [22].

In this paper we shall use the formalism developed in our earlier work [20] to analyze the nearhorizon geometry of a large class of black holes, including that for the BTZ. Unlike [20, here we use an arbitrary frequency mode of a massless scalar field to probe the near-horizon region. The central charge of the near-horizon CFT is obtained as a continuous variable. It is determined by the coefficient of the inverse square term in the near-horizon operator, which depends on the black hole parameters and the frequency of the probe. In order that our near-horizon CFT admits a central charge $c=24$ requires a quantization condition. This condition fixes the scalar field frequency in terms of the black hole parameters. Witten has suggested that the AdS/CFT dual of the BTZ black hole is the $c=24$ conformal field theory of FLM, which describes only gravitational degrees of 
freedom and is conjectured to be unique. In our analysis there is no AdS/CFT correspondence, but we find the possibility of a $c=24 \mathrm{CFT}$ for the BTZ. The uniqueness conjecture then implies that the $c=24$ CFT obtained in our analysis should also be identified with the FLM construction. In addition, we explicitly show that a large class of black holes contain the same near-horizon conformal structure as that for the BTZ, all of which admit a $c=24$ CFT in the near-horizon region. From the uniqueness conjecture it follows that all these CFT's should also be identified with the FLM construction. The $c=24$ FLM CFT has the largest degeneracy of all allowed finite CFT's and hence provides a bound on the black hole entropy. The emergence of this possibility in various backgrounds considered here is consistent with the fact that the BTZ black hole appears in the near-horizon region of many black holes [23, 24, 25, 26, 27, 28, 29, 30].

This paper is organized as follows. In Section 2, we study the dynamics of the scalar field probe in the near-horizon region for the BTZ black hole. In Section 3, this analysis is generalized to include a wide class of black holes. Different black hole near horizon structures and the near horizon KG operator including the Gauss-Bonnet case obtained from string derived gravity is discussed in the Appendix. It is shown that the near-horizon dynamics of the scalar probe is governed by an operator which has a universal form. In Section 4, the algebraic properties of the near-horizon Klein-Gordon operator is studied which reveals the existence of an underlying conformal algebra consisting of a semi-direct product of the Virasoro algebra and the algebra of the shift operators. In Section 5, we study the representations of this algebra and obtain a universal form of the central charge. Section 6 discusses the connection of the central charge to the black hole entropy in the context of the FLM CFT obtained by Witten. Section 7 concludes the paper with some discussions.

\section{Scalar field probe of the near-horizon geometry of BTZ black hole}

In $2+1$ dimensions, a BTZ black hole of mass $M$ and spin $J$ is given by the metric [10, 11]

$$
d s^{2}=-\left(-M+\frac{r^{2}}{l^{2}}+\frac{J^{2}}{4 r^{2}}\right) d t^{2}+\left(-M+\frac{r^{2}}{l^{2}}+\frac{J^{2}}{4 r^{2}}\right)^{-1} d r^{2}+r^{2}\left(d \phi-\frac{J}{2 r^{2}} d t\right)^{2}
$$

where $r$ and $\phi$ denote the radial and angular coordinates on the plane and $t$ denotes the time. This metric satisfies vacuum Einstein equations in $2+1$ dimensions, with a negative cosmological constant $\Lambda=-1 / l^{2}$. The outer and inner horizons denoted by $r_{ \pm}$respectively are given by

$$
r_{ \pm}^{2}=\frac{M l^{2}}{2}\left(1 \pm \sqrt{1-\frac{J^{2}}{M^{2} l^{2}}}\right)
$$

In the present approach, a massless scalar field is used as a probe of the near-horizon geometry. The Klein-Gordon (KG) equation for a massless scalar field $\psi$ in a general space-time with a metric $g_{\mu \nu}$, where $\mu, \nu$ run over the space-time indices, is given by

$$
\frac{1}{\sqrt{-g}} \partial_{\mu}\left(\sqrt{-g} g^{\mu \nu} \partial_{\nu} \psi\right)=0
$$


For the BTZ metric (2.1), using the ansatz $\psi(r, t, \phi)=R(r) e^{-i \omega t+i k \phi}$, one gets the radial equation as 31 .

$$
\partial_{r}^{2} R(r)+\left(-\frac{1}{r}+\frac{2 r}{r^{2}-r_{-}^{2}}+\frac{2 r}{r^{2}-r_{+}^{2}}\right) \partial_{r} R(r)+N^{-4}\left(\omega^{2}-\frac{J \omega k}{r^{2}}+\frac{B k^{2}}{r^{2}}\right) R(r)=0
$$

where $N^{2}=\frac{1}{l^{2} r^{2}}\left(r^{2}-r_{-}^{2}\right)\left(r^{2}-r_{+}^{2}\right)$ and $B=M-\frac{r^{2}}{l^{2}}$. The periodicity of the coordinate $\phi$ in the BTZ construction leads to the quantization condition $k \in \mathbf{Z}$.

Our main interest is to probe the region near the outer horizon of this black hole. To this end, we define a near-horizon coordinate $x \in[0, \infty)$ as

$$
x=r-r_{+} .
$$

In the near-horizon region, the function $N^{2}$ takes the form

$$
N^{2}(x)=A x, \quad A=\frac{2}{l^{2} r_{+}}\left(r_{+}^{2}-r_{-}^{2}\right)
$$

We now define a new radial wave-function $\chi(r) \equiv \sqrt{x} R(r)$, in terms of which the near-horizon form of the $\mathrm{KG}$ equation (2.4) is given by

$$
H \chi(r) \equiv\left[-\frac{d^{2}}{d x^{2}}+\frac{a}{x^{2}}\right] \chi(r)
$$

where

$$
a=-\left[\frac{1}{4}+\frac{\tilde{\omega}^{2}}{A^{2}}\right], \quad \tilde{\omega}^{2}=\omega^{2}-\frac{J \omega k}{r_{+}^{2}}+\frac{M k^{2}}{r_{+}^{2}}-\frac{k^{2}}{l^{2}}
$$

In (2.7), $H$ denotes the near-horizon KG operator for the BTZ black hole. The parameter $a$ contains the information specific to the geometry. Below we shall show that the near-horizon KG operator for a large class of black holes has the same form as in (2.7) with different values of the parameter $a$.

\section{Universal near-horizon geometry for a large class of black holes}

Consider a metric in $D$ space-time dimensions given by

$$
d s^{2}=-f(r) d t^{2}+\frac{d r^{2}}{f(r)}+r^{2} d \Omega_{D-2}^{2}
$$

where $f(r)$ is a function of the radial variable $r$ and $d \Omega_{D-2}^{2}$ is the metric on unit $S^{D-2}$. The form of the functions $f(r)$ will depend on the choice of the specific black hole.

As before, we wish to probe the near-horizon geometry of the black hole using a massless scalar field. To that end, we again consider the dynamics of a scalar field $\psi$ in the above general background. Using the ansatz $\psi(t, r, \Omega)=e^{-i \omega t} R(r) Y_{l m}(\Omega)$, the KG equation for the general background (3.1) can be written as

$$
\frac{d^{2} R(r)}{d r^{2}}+\frac{\left(f(r) r^{D-2}\right)^{\prime}}{f(r) r^{D-2}} \frac{d R(r)}{d r}+\frac{\omega^{2} R(r)}{f^{2}(r)}-\frac{1}{f(r)}\left(\frac{l(l+D-3)}{r^{2}}\right) R(r)=0
$$


where the prime denotes the derivative with respect to the radial coordinate.

As in the case for BTZ, we now define a near-horizon coordinate $x \equiv r-r_{h}$, where $r_{h}$ denotes the event horizon and $x \in[0, \infty)$. We shall show below that the near-horizon form of the function $f$ for a large class of black holes is given by

$$
f(x) \sim A x[1+\mathcal{O}(x)]
$$

where $A$ is a constant which depends on parameters defining the black hole geometry. For the moment we proceed with the form of $f$ given in (3.3). In terms of a new field $\chi \equiv \sqrt{x} R(r)$, the KG equation in the near-horizon region takes the form

$$
H \chi=\left[-\frac{d^{2}}{d x^{2}}+\frac{a}{x^{2}}\right] \chi,
$$

where

$$
a=-\left[\frac{1}{4}+\frac{\omega^{2}}{A^{2}}\right] .
$$

The above analysis thus shows that for the entire class of metrics (3.1) satisfying the condition (3.3), the near-horizon KG operator $H$ for a massless scalar has a universal form given by

$$
H=-\frac{d^{2}}{d x^{2}}+\frac{a}{x^{2}}
$$

The structure of $H$ here is the same as that for the BTZ black hole as given by (2.7). The constant $a$ depends on the frequency of the probe and on the geometric details and distinguishes between the various black holes. For real and nonzero frequency of the scalar probe the constant $a$ is real and satisfies the condition $a<-\frac{1}{4}$.

The above analysis shows that for a very large class of black holes, the near-horizon KG operator for a massless scalar has a universal form. This result is somewhat surprising as the black holes have quite different geometric properties. We have discussed different black hole metrics in detail in the appendix and it can be seen from the discussion in the appendix that apart from asymptotically flat backgrounds, our analysis includes black holes with both signs of the cosmological constant. It also includes the Gauss-Bonnet case which is obtained from string derived gravity going beyond the usual Einstein-Hilbert action. In a later section we shall comment on the possible common link between the near-horizon structures of these varied class of black holes with that for BTZ. Before that we shall study the algebraic properties of the near-horizon KG operator.

\section{Algebraic properties of the near-horizon KG operator}

We have seen from the previous section that for a large class of black holes, the KG operator in the near-horizon region has a universal form given by (3.6). In this section we shall use algebraic techniques to study the properties of $H$. 
Following our earlier work [20, 32], the operator $H$ can be decomposed as

$$
H=C_{+} C_{-}, \quad \text { where } \quad C_{ \pm}= \pm \frac{d}{d x}+\frac{b}{x},
$$

and

$$
b=\frac{1}{2} \pm \frac{\sqrt{1+4 a}}{2} .
$$

For the present cases of interest, $a<-\frac{1}{4}, b$ is complex and $C_{+}$and $C_{-}$are not formal adjoints of each other. Following [20] we define the operators

$$
\begin{aligned}
L_{n} & =-x^{n+1} \frac{d}{d x}, \quad n \in \mathbf{Z}, \\
P_{m} & =\frac{1}{x^{m}}, \quad m \in \mathbf{Z}
\end{aligned}
$$

Using Equation (4.3) and (4.4), the operators $C_{ \pm}$and $H$ can be written as

$$
\begin{aligned}
C_{ \pm} & =\mp L_{-1}+b P_{1}, \\
H & =\left(-L_{-1}+b P_{1}\right)\left(L_{-1}+b P_{1}\right) .
\end{aligned}
$$

The operators $L_{m}, P_{m}$ and $H$ satisfy the commutation relations

$$
\begin{aligned}
{\left[P_{m}, P_{n}\right] } & =0, \\
{\left[L_{m}, P_{n}\right] } & =n P_{n-m}, \\
{\left[L_{m}, L_{n}\right] } & =(m-n) L_{m+n}+\frac{c}{12}\left(m^{3}-m\right) \delta_{m+n, 0}, \\
{\left[P_{m}, H\right] } & =m(m+1) P_{m+2}+2 m L_{-m-2}, \\
{\left[L_{m}, H\right] } & =2 b(b-1) P_{2-m}-(m+1)\left(L_{-1} L_{m-1}+L_{m-1} L_{-1}\right) .
\end{aligned}
$$

Note that Equation (4.9) describes a Virasoro algebra with central charge $c$, while the algebra of the generators defined in Equation (4.3) would lead to $\left[L_{m}, L_{n}\right]=(m-n) L_{m+n}$. However, this algebra admits a non-trivial central extension $c$ and in any of its unitary irreducible highest weight representation $c \neq 0$ [33]. That is why the central charge has been explicitly included in (4.9)).

Equations (4.7-4.9) describe the semidirect product of the Virasoro algebra with an Abelian algebra satisfied by the shift operators $P_{m}$ [34]. This semidirect product algebra will be denoted by $\mathcal{M}$ in the rest of this paper. Note that $L_{-1}$ and $P_{1}$ are the only generators that appear in $H$. Starting with these two generators, and using (4.10) and (4.11), we see that the only operators which appear are the Virasoro generators with negative index (except $L_{-2}$ ) and the shift generators with positive index. Thus, $L_{m}$ with $m \geq 0$ and $P_{m}$ with $m \leq 0$ do not appear in the above expressions. In the next section, we will discuss how these quantities are generated.

We also note that the operator $H$ is not an element of $\mathcal{M}$ but belongs to the corresponding enveloping algebra. This is due to the fact that the right hand side of Equation (4.11) contains product of Virasoro generators. While such products are not elements of the algebra, they do belong to the corresponding enveloping algebra. 


\section{Representation theory}

The representation theory of $\mathcal{M}$ is well known in the literature [34]. Below we briefly recall certain aspects of this theory relevant for our analysis.

Consider the space $V_{\alpha, \beta}$ of densities containing elements of the form $P(x) x^{\alpha}(d x)^{\beta}$. Here, $\alpha, \beta$ are complex numbers and $P(x)$ is an arbitrary polynomial in $x$ and $x^{-1}$. Note that $x$ is now treated as a complex variable and the algebra $\mathcal{M}$ remains unchanged even when $x$ is complex. $V_{\alpha, \beta}$ carries a representation of the algebra $\mathcal{M}$. The space $V_{\alpha, \beta}$ is spanned by a set of basis vectors $\omega_{m}=x^{m+\alpha}(d x)^{\beta}$, where $m \in \mathbf{Z}$. It can be shown that the Virasoro generators and the shift operators have the following action on the basis vectors $\omega_{m}$ [34]

$$
\begin{aligned}
& P_{n}\left(\omega_{m}\right)=\omega_{m-n}, \\
& L_{n}\left(\omega_{m}\right)=-(m+\alpha+\beta+n \beta) \omega_{n+m} .
\end{aligned}
$$

The representation $V_{\alpha, \beta}$ is reducible if $\alpha \in \mathbf{Z}$ and if $\beta=0$ or 1 ; otherwise it is irreducible.

The requirement of unitarity of the representation $V_{\alpha, \beta}$ leads to several important consequences. In any unitary representation of $\mathcal{M}$, the Virasoro generators must satisfy the condition $L_{-m}^{\dagger}=L_{m}$. In the previous section, we saw that $L_{-2}$ and $L_{m}$ for $m \geq 0$ did not appear in the algebraic structure generated by the basic operators appearing in the factorization of $H$. However, the requirement of a unitary representation now leads to the inclusion of $L_{m}$ for $m>0$. The remaining generators now appear through appropriate commutators, thus completing the algebra $\mathcal{M}$.

Unitarity also constrains the parameters $\alpha$ and $\beta$, which must satisfy the conditions

$$
\begin{aligned}
& \beta+\bar{\beta}=1, \\
& \alpha+\beta=\bar{\alpha}+\bar{\beta},
\end{aligned}
$$

where $\bar{\alpha}$ denotes the complex conjugate of $\alpha$. The central charge $c$ in the representation $V_{\alpha, \beta}$ is then given by 34

$$
c(\beta)=-12 \beta^{2}+12 \beta-2 .
$$

Next we study the quantum properties of the Klein-Gordon operator $H$ in the near horizon region of the black holes. In particular, we shall analyze the eigenvalue equation

$$
H|\Psi\rangle=E|\Psi\rangle
$$

using the representations of the algebra $\mathcal{M}$ and we shall find the labels $\alpha$ and $\beta$ of the representation of $\mathcal{M}$ in terms of the black hole parameters.

We choose an ansatz for the wave function $|\Psi\rangle$ given by $|\Psi\rangle=\sum_{n=0}^{\infty} c_{n} \omega_{n}$. The indicial equation arising out from the substitution of the above ansatz in the eigenvalue equation (5.6) is given by

$$
\alpha=b, \text { or }(1-b)
$$


As discussed before, for nonzero real frequency of the probe, the constant $a$ is real and satisfies the condition $a<-\frac{1}{4}$. Thus, in the general case, we can use the parametrization

$$
a=-\frac{1}{4}-\mu^{2}
$$

The information about the black hole geometry is contained in the parameter $\mu \in R$. Using (5.8) in (4.2), we obtain

$$
b=\frac{1}{2} \pm i \mu
$$

Equations (5.7) and (5.9) determine the label $\alpha$ in the representation of $\mathcal{M}$, whose allowed values are given by

$$
\alpha=\frac{1}{2} \pm i \mu, \quad \text { or } \quad \alpha=-\frac{1}{2} \mp i \mu \text {. }
$$

Using (5.3) and (5.4), the other parameter $\beta$ in the representation of $\mathcal{M}$ is determined in terms of $\alpha$ as

$$
\beta=\frac{1}{2}-\operatorname{Im} \alpha
$$

The central charge $c$ in any representation of $\mathcal{M}$ depends only on the value of $\beta$. Using (5.5), (5.10) and (5.11), the central charge is obtained as

$$
c=1+12 \mu^{2}
$$

This expression of the central charge has two contributions. The first part is a constant 1, which is independent of the black hole parameters. The second part depends on $\mu$, which contains information about the black hole parameters. In our framework, we associate the quantity $c_{b h} \equiv 12 \mu^{2}$ with the contribution to the central charge due to the black hole, as detected by the scalar field probe. In the subsequent analysis we shall focus on $c_{b h}$ only.

\section{Central charge and black hole entropy}

The relation between CFT and black hole entropy has been extensively discussed in the literature [1, 2, 3]. In the Chern-Simons approach to $2+1$ gravity with a negative cosmological constant [13, 14], the method of Brown and Henneaux [12 predicts a continuously varying central charge. This is a purely classical result where nothing in this analysis puts any constraint on the central charge. However, motivated by the AdS/CFT correspondence, Witten [9] argued that the central charge cannot vary continuously, but must be quantized. In the Chern-Simons approach to BTZ, this assumption leads to a central charge given by $c=24 k$, where $k$ is the coefficient in front of the Chern-Simons action. The constant $k$, for a suitable choice of the gauge group, is quantized as a positive integer. Assuming $k=1$ for the moment, the central charge has the value $c=24$. Of all the holomorphic CFT's associated with $c=24$, there is only one which corresponds to pure gravity. Such 
a CFT model was explicitly constructed by Frenkel, Lepowsky and Meurman (FLM)[15], who also conjectured that it would be unique. This model admits a huge symmetry, given by the Fischer-Griess monster group. Using the FLM representation and exploiting its uniqueness, Witten obtained the entropy of the quantum BTZ black hole which agrees reasonably well with the Bekenstein-Hawking entropy.

In our formalism, the central charge associated with the black hole degrees of freedom is given by $c_{b h}=12 \mu^{2}$. From the pure classical gravity point of view, there is no constraint on this central charge. However, motivated by the same logic of Witten, we also demand quantization of the central charge for the BTZ. In particular, we impose the condition $\mu^{2}=2 n$, where $n$ is a positive integer. Then, for $n=1$, we have a CFT with central charge equal to 24 . The FLM construction is conjectured to give a unique CFT for pure gravity with $c=24$. Thus, the above quantization condition together with the uniqueness conjecture for the FLM construction implies that the near-horizon CFT for the BTZ black hole in our approach as well is given by the FLM CFT. Consequently, the entropy calculation of Witten for the BTZ black hole based on the monster symmetry of the FLM CFT would apply in our framework too. Let us note that our argument is valid only with the quantization condition $\mu^{2}=2 n$. The quantity $\mu^{2}$ depends on the scalar field frequency and also on the geometric parameters defining the BTZ black hole. This condition implies that the scalar field frequency is quantized and that the scale of the frequency is set by the geometric parameters of the BTZ. These consequences are completely natural and such a quantization of the frequency is also in qualitative agreement with the brick wall approach to black hole entropy [35, 36].

The present analysis also indicates that the near-horizon conformal structure for the BTZ is shared by a wide class of black holes. In particular, the near-horizon KG operator (3.6) and the central charge (5.12) have the same universal form for all the black holes considered here. For this larger class, we can again identify $12 \mu^{2}$ in the central charge as the contribution associated to the black hole geometry, as this part depends explicitly on the black hole parameters. In string theory it is well known that a large class of black holes, including the Schwarzschild case, contains a BTZ factor as a part of their near-horizon geometry [5, 23, 24, 25, 26, 27, 28, 29, 30]. The result that a large class of black holes share a universal near-horizon conformal structure identical to that of the BTZ is thus consistent with the observations from string theory.

The existence of a BTZ factor in the near-horizon region for a large class of black holes has been very useful in calculating the black hole entropy in string theory [28, 30]. Motivated by this observation, we can conjecture that the central charge for the entire class of black holes that share the same near-horizon conformal structure as that for the BTZ should also be subject to a similar quantization. Imposition of the same quantization condition as in BTZ leads to $c_{b h}=24$ in each of these cases. Moreover, the scalar field frequency for each background would now be quantized, where the scales in the frequencies would be set by the respective black hole parameters. Since we are dealing with pure gravity, the FLM construction would provide the unique CFT in each of these cases, leading to a universal form of entropy for the entire class. This presents an apparent puzzle, as surely the Bekenstein-Hawking entropy for a Schwarzschild black hole is not going to be the same as that for BTZ. This can be resolved by noting that the near-horizon geometry for a general black hole contains factors other than the BTZ, which can also contribute to the entropy. Thus in our analysis, the BTZ entropy contribution provides a lower bound to the entropy of this entire class of 
black holes.

\section{Conclusion}

In this paper we have analyzed the near-horizon conformal structure for a large class of black holes, using a scalar field as a probe of the geometry. The class of backgrounds considered here includes black holes in various dimensions, with or without cosmological constant. We have also considered Gauss-Bonnet black hole, which is obtained from string derived gravity by going beyond the usual Einstein-Hilbert action of general relativity.

The analysis presented here reveals certain universal characteristics in the near-horizon conformal structure. In particular, the near-horizon KG operator and the central charge of the associated Virasoro algebra has the same universal form for the entire class of black holes discussed here. Our analysis is consistent with the observation in string theory that a large class of black holes contains a BTZ part as their near-horizon geometry. The BTZ entropy has recently been obtained by Witten using the FLM construction. Although we do not have an AdS/CFT correspondence, our analysis for the BTZ black hole is consistent with Witten's observations after a suitable quantization condition is imposed. Furthermore, assuming that such a quantization can be imposed for the other cases considered here, it appears plausible that the FLM CFT provides a universal contribution to the entropy for a large class of black holes. The actual entropy would depend on other factors as well, but the universal part in our approach is determined by the FLM model, which provides a lower bound to the entropy of this wide class of black holes. For any given background, the assumed condition on the central charge also leads to the quantization of the scalar field frequency, where the scale is set by the parameters defining the respective black hole.

In this paper, although black holes with positive cosmological constants have been analyzed, the physical interpretation in such cases are subject to usual ambiguities associated with de-Sitter spaces [37, 38]. It would be interesting to see if some of those ambiguities can be resolved in the framework of the near-horizon CFT discussed here. We have also not discussed the extremal black holes, which would also be of interest.

\section{Appendix}

In this appendix we shall give examples of different black hole metrics for which the function $f$ has the form as given in (3.3). We shall first discuss the examples where the cosmological constant vanishes

\section{Asymptotically flat black hole geometries}

\section{Schwarzschild black hole:}

The metric function $f(r)$ is given by

$$
f(r)=1-\frac{2 M}{r^{D-3}}
$$


The event horizon, determined by $f(r)=0$, is located at

$$
r_{h}=(2 M)^{\frac{1}{D-3}}
$$

so that $f(r) \geq 0$ for $r \geq r_{h}$ and the parameter $M$ is always positive. Now, the form of the metric near the horizon is given by

$$
f(x)=A x, \text { where } A=\frac{2 M(D-3)}{r_{h}^{D-2}} .
$$

Here $A$ is a constant for a particular $M$.

\section{Reissner Nordström black hole:}

The function $f(r)$ has the following form

$$
f(r)=1-\frac{2 M}{r^{D-3}}+\frac{Q^{2}}{r^{2 D-6}}
$$

and the event horizon corresponds to

$$
r_{h}^{ \pm}=\left(M \pm \sqrt{M^{2}+Q^{2}}\right)^{\frac{1}{D-3}}
$$

Here, we are mainly interested in the outer horizon, $r_{h}^{+}$, since all our calculations will eventually involve the near-horizon coordinate and here 'near-horizon' implies near the outer horizon. In this

case $f(r) \geq 0$ for $r \geq r_{h}^{+}$. This essentially leads to the constraint $Q^{2} \leq M^{2}$. Now, the form of the metric near the horizon is given by

$$
f(x)=A x, \text { where } A=\left[\frac{2 M(D-3)}{r_{h}^{+D-2}}-\frac{Q^{2}(2 D-6)}{r_{h}^{+2 D-5}}\right] .
$$

\section{Uncharged Gauss-Bonnet black hole:}

In $D$ space-time dimension $(D \geq 5)$, the metric for spherically symmetric asymptotically flat GaussBonnet black hole of mass $M$ is given by Eqn. (3.1), where $f(r)$ has the form [39]

$$
f(r)=1+\frac{r^{2}}{2 \alpha}-\frac{r^{2}}{2 \alpha} \sqrt{1+\frac{8 \alpha M}{r^{D-1}}}
$$

where

$$
\alpha=16 \pi G_{D}(D-3)(D-4) \alpha^{\prime}
$$


For $\alpha^{\prime}>0$, this black hole admits only a single horizon [39]. The horizon $r=r_{h}$ is determined by the real positive solution of the equation

$$
r_{h}^{D-3}+\alpha r_{h}^{D-5}=2 M
$$

In terms of the near-horizon coordinate the function $f(r)$ becomes

$$
f(x)=A x, \quad \text { where } A=\left[\frac{r_{h}^{2}(D-3)+\alpha(D-5)}{r_{h}\left(r_{h}^{2}+2 \alpha\right)}\right] .
$$

\section{Asymptotically non-flat black hole geometries:}

\section{Schwarzschild-AdS black hole:}

The metric in this case is given by

$$
f(r)=1-\frac{2 M}{r^{D-3}}-\Lambda r^{2}
$$

where $\Lambda<0$ and $M>0$. There is an event horizon defined by $f(r)=0$ and given by the unique, real and positive root of

$$
|\Lambda| r^{D-1}+r^{D-3}-2 M=0
$$

There is no cosmological horizon here. The form of the metric near the horizon is given by

$$
f(x)=A x, \text { where } A=\left[\frac{2 M(D-3)}{r_{h}^{D-2}}-2 r_{h} \Lambda\right] .
$$

\section{Reissner Nordström-AdS black hole:}

The function $f(r)$ has the following form

$$
f(r)=1-\frac{2 M}{r^{D-3}}+\frac{Q^{2}}{r^{2 D-6}}-\Lambda r^{2}, \quad \Lambda<0
$$

The event horizons are given by the solutions of the equation

$$
|\Lambda| r^{2 D-4}+r^{2 D-6}-2 M r^{D-3}+Q^{2}=0,
$$

and there is no cosmological horizon. This equation has two real positive zeroes which gives the inner and outer horizons of the black hole. But, $f(r) \geq 0$ for $r \geq r_{h}^{+}$. Here also we are interested in the 
outer horizon. Here $Q^{2}<M^{2}$ and $\Lambda<0$. The function $f(r)$ in the near horizon coordinate has the form

$$
f(x)=A x, \text { where } A=\left[\frac{2 M(D-3)}{r_{h}^{+D-2}}-\frac{Q^{2}(2 D-6)}{r_{h}^{+2 D-5}}-2 r_{h}^{+} \Lambda\right]
$$

\section{Schwarzschild-dS black hole:}

The metric has the same form as of Schwarzschild-AdS black hole, except the fact that the cosmological constant $\Lambda>0$ here. There is both an event horizon $r_{h}$ as well as a cosmological horizon $r_{c}$ which are given by the real positive roots of

$$
\Lambda r^{D-1}-r^{D-3}+2 M=0 .
$$

This equation has two real positive zeroes corresponding to the event and the cosmological horizon. The function $f(r) \geq 0$ for $r_{h} \leq r \leq r_{c}$. We are interested in the region outside the event horizon only.

The form of the constant $A$ is given by $A=\left[\frac{2 M(D-3)}{r_{h}^{D-2}}-2 r_{h} \Lambda\right]$, i.e. it is same as that of Schwarzschild AdS black hole except here the cosmological constant is positive.

\section{Reissner Nordström-dS black hole:}

The metric here also looks like the same as of RN-AdS case except the cosmological constant being positive here. There are two event horizons now and a cosmological horizon given by the zeroes of

$$
\Lambda r^{2 D-4}-r^{2 D-6}+2 M r^{D-3}-Q^{2}=0 .
$$

This equation now has three real positive roots corresponding to inner and outer event horizons and a cosmological horizon. Here $f(r) \geq 0$ for $r_{h}^{+} \leq r \leq r_{c}$. Here also the structure of $A$ is same as that of the RN-AdS case but with positive cosmological constant.

\section{References}

[1] A. Strominger, JHEP 9802, 009 (1998).

[2] S. Carlip, Phys. Rev. D55, 878 (1997); Class. Quant. Grav. 15, 3609 (1998); Class. Quant. Grav. 16, 3327 (1999); Phys. Rev. Lett. 82, 2828 (1999); Nucl. Phys. Proc. Suppl. 18, 10 (2000); Phys. Rev. Lett. 88, 241301 (2002); Class. Quant. Grav. 22, R85 (2005); J. Phys. Conf. Ser. 67, 012022 (2006); arXiv:0705.3024 [gr-qc].

[3] S. N. Solodukhin, Phys. Lett. B454, 213 (1999); T. R. Govindarajan, R. K. Kaul and V. Suneeta, Class. Quant. Grav. 18 2877(2001); Class. Quant. Grav. 19, 4195 (2002); G. A. S. Dias and J. P. S. Lemos, Phys. Rev. D74, 044024 (2006). 
[4] J. M. Maldacena, Adv. Theor. Math. Phys. 2, 231 (1998) [Int. J. Theor. Phys. 38, 1113 (1999).]

[5] O. Aharony, S. S. Gubser, J. M. Maldacena, H. Ooguri and Y. Oz, Phys. Rept. 323, 183 (2000).

[6] G. 't Hooft, arXiv:gr-qc/9310026.

[7] L. Susskind, J. Math. Phys. 36, 6377 (1995).

[8] L. Susskind and E. Witten, arXiv:hep-th/9805114.

[9] E. Witten, arXiv:0706.3359v1 [hep-th].

[10] M. Banados, C. Teitelboim and J. Zanelli, Phys. Rev. Lett. 69, 1849 (1992).

[11] M. Banados, M. Henneaux, C. Teitelboim and J. Zanelli, Phys. Rev. D48, 1506 (1993).

[12] J. D. Brown and M. Henneaux, Commun. Math. Phys. 104, 207 (1986).

[13] A. Achúcarro and P. Townsend, Phys. Lett. B180, 89 (1986).

[14] E. Witten, Nucl. Phys. B311, 46 (1988).

[15] I. B. Frenkel, J. Lepowsky and A. Meurman, Proc. Natl. Acad. Sci. USA 81, 3256 (1984).

[16] M. P. Tuite, Commun. Math. Phys. 166, 495 (1995).

[17] G. Kang, J. Koga and M.-I. Park, Phys. Rev. D70, 024005 (2004).

[18] M. Cvitan, S. Pallua and P. Prester, Phys. Lett. B555, 248 (2003); M. Cvitan, S. Pallua and P. Prester, Phys. Lett. B571, 217 (2003).

[19] H. E. Camblong and C. R. Ordóñez, Phys. Rev. D71, 104029 (2005).

[20] D. Birmingham, K. S. Gupta and S. Sen, Phys. Lett. B 505, 191 (2001).

[21] K. S. Gupta and S. Sen, Phys.Lett. B526, 121 (2002); K. S. Gupta, hep-th/0204137.

[22] K. Srinivasan and T. Padmanabhan, Phys. Rev. D60 024007 (1999).

[23] J. M. Maldacena and A. Strominger, JHEP 9812, 005 (1998).

[24] D. Birmingham, I. Sachs and S. Sen, Phys. Lett. B424, 275 (1998).

[25] K. Sfetsos and K. Skenderis, Nucl. Phys. B517, 179 (1998).

[26] M. Cvetic and F. Larsen, Phys. Rev. Lett. 82, 484 (1999).

[27] Y. Satoh, Phys. Rev. D59, 084010 (1999).

[28] A. W. Peet, arXiv:hep-th/0008241. 
[29] K. S. Gupta and S. Sen, Phys. Lett. B646, 265 (2007).

[30] Ashoke Sen, arXiv:0708.1270.

[31] D. Birmingham and S. Sen, Int. J. Mod. Phys. D10, 833 (2001).

[32] K. S. Gupta and S. G. Rajeev, Phys. Rev. D48, 5940 (1993).

[33] P. Goddard and D. Olive, Int. Jour. Mod. Phys. A1, 303 (1986).

[34] Bombay Lectures on Highest Weight Representations of Infinite Dimensional Lie Algebras, V.G.Kac and A.K.Raina (World Scientific, Singapore, 1987).

[35] G. 't Hooft, Nucl. Phys. B256, 727 (1985).

[36] K. S. Gupta and S. Sen, Mod. Phys. Lett. A18, 1463 (2003).

[37] E. Witten, arXiv: hep-th/0106109.

[38] N. Goheer, M. Kleban and L. Susskind, JHEP 0307, 056 (2003).

[39] D. G. Boulware and S. Deser, Phys. Rev. Lett. 55, 2656 (1985). 